\title{
Rosuvastatin blocks allergic airway vagal hypertonia via reversed central CD73 downregulation in rats
}

\author{
Hong Chen ${ }^{1}$, Ming Zeng ${ }^{1}$, Yun Lin ${ }^{1}$, Jialun Jiang${ }^{1}$, Yi Yang ${ }^{1}$, Yonghua Chen ${ }^{1}$, Chunmei \\ $\mathrm{Xia}^{1}$, Jin Wang ${ }^{1}$, and Jijiang Wang ${ }^{1}$ \\ ${ }^{1}$ Fudan University School of Basic Medical Sciences
}

July 30, 2020

\begin{abstract}
Background In asthma, the decrease of pulmonary function and occurrence of pulmonary inflammation are largely attributed to the increase of airway vagal activity, of which the genesis involves disrupted degradation of central extracellular ATP to adenosine due to decreased expression and activity of ecto-5'-nucleotidase (CD73). Meanwhile, the therapeutic use of statins reportedly is able to alleviate asthma; however, the mechanisms remain unclear. This study test whether rosuvastatin is able to attenuate the downregulation of central CD73 and, subsequently, attenuate the increase of airway vagal activity in the rat model of allergic asthma. Methods An experimental rat model of allergic asthma was prepared using ovalbumin. During the sensitization period, ovalbumin was inhaled alone or in combination with rosuvastatin. Plethysmographic measurement of pulmonary function was used to evaluate airway vagal activity; molecular biological assay was used to examine the expression and activity of medullary CD73 and the expression of eosinophil cationic protein 1 (ECP1) in the lungs. Results In ovalbuminsensitized rats, the decreases in the expression and activity of medullary CD73 and the increases in the expression of ECP1 in the lungs and ATP concentration in cerebral spinal fluid were completely reversed by inhaled rosuvastatin, so was the increase of airway vagal activity manifested by the atropine-sensitive increase of airway resistance and decrease of airway compliance. Conclusions In the rat model of allergic asthma, inhaled rosuvastatin reverses the decreases in the expression and activity of brainstem CD73, which prevents pulmonary function decrease via an abolished increase of airway vagal activity.
\end{abstract}

Title: Rosuvastatin blocks allergic airway vagal hypertonia via reversed central CD73 downregulation in rats

Running title : Statin improvement of pulmonary function in asthma

Authors: Hong Chen\#; Ming Zeng\#; Yun Lin, Jialun Jiang, Yi Yang; Yonghua Chen, Ph.D; Chunmei Xia, Ph.D; Jin Wang, Ph.D Jijiang Wang, Ph.D*

\#, these authors contribute equally to this study.

Affiliations: Department of Physiology and Pathophysiology, Fudan University School of Basic Medical Sciences.

\section{Author Contributions}

Jijiang Wang designed the study; Hong Chen, Ming Zeng, Yun Lin, Jialun Jiang and Yi Yang performed the experiments and analyzed the data; Yonghua Chen, Chunmei Xia and Jin Wang helped interpretation of the data. All of the authors were involved in preparation and improvement of the manuscript.

*Corresponding author: Jijiang Wang, Ph.D: Department of Physiology and Pathophysiology, Fudan University School of Basic Medical Sciences. 130 Dong'an Rd., 207 seventh building, west campus, Shanghai 200032, China. Tel: +8621-54237405. Email:wangjj@shmu.edu.cn 
Funding Sources: This study was sponsored by the NSFC (National Natural Science Foundation of China) grants 81970002 and 81770003 to J Wang.

Three figures; 248 words in Abstract; 559 words in Introduction; 1054 words in Discussion

\section{Abstract}

\section{Background}

In asthma, the decrease of pulmonary function and occurrence of pulmonary inflammation are largely attributed to the increase of airway vagal activity, of which the genesis involves disrupted degradation of central extracellular ATP to adenosine due to decreased expression and activity of ecto-5'-nucleotidase (CD73). Meanwhile, the therapeutic use of statins reportedly is able to alleviate asthma; however, the mechanisms remain unclear. This study test whether rosuvastatin is able to attenuate the downregulation of central CD73 and, subsequently, attenuate the increase of airway vagal activity in the rat model of allergic asthma.

\section{Methods}

An experimental rat model of allergic asthma was prepared using ovalbumin. During the sensitization period, ovalbumin was inhaled alone or in combination with rosuvastatin. Plethysmographic measurement of pulmonary function was used to evaluate airway vagal activity; molecular biological assay was used to examine the expression and activity of medullary CD73 and the expression of eosinophil cationic protein 1 (ECP1) in the lungs.

\section{Results}

In ovalbumin-sensitized rats, the decreases in the expression and activity of medullary CD73 and the increases in the expression of ECP1 in the lungs and ATP concentration in cerebral spinal fluid were completely reversed by inhaled rosuvastatin, so was the increase of airway vagal activity manifested by the atropinesensitive increase of airway resistance and decrease of airway compliance.

\section{Conclusions}

In the rat model of allergic asthma, inhaled rosuvastatin reverses the decreases in the expression and activity of brainstem CD73, which prevents pulmonary function decrease via an abolished increase of airway vagal activity.

Key words: asthma; asthma treatment; basic mechanisms.

Abstract word count: 248 .

\section{Introduction}

Asthma produces heavy social and financial burdens, making it one of the major non-communicable diseases worldwide (1). Asthma manifests as chronic airflow obstruction, with characteristic chronic airway inflammation and hypersensitivity/hyperresponsiveness. Increased airway vagal activity is well known to play important roles in the asthmatic constriction, mucous secretion, inflammation and remodeling of the airway (2-5). Accordingly, inhaled muscarinic antagonists, alone or in combination with glucocorticoids and/or $\beta_{2}{ }^{-}$ adrenergic agonists, have become the common therapy for asthma clinically, especially in its severe attacks (6). In patients with obstinate severe asthma symptoms, high selective vagotomy of hilus pulmonis has become a choice of treatment (7). Regretfully, none of these inhaled anti-asthma drugs is able to eradicate the asthmatic increase of airway vagal activity; and surgical treatment is less acceptable clinically due to the invasiveness. Therefore, it is still urgent to find therapies that are more specifically against the genesis of the asthmatic increase of airway vagal activity.

In a rat model of allergic asthma, we recently proved that the decreases in the expression and activity of central ecto-5'-nucleotidase (CD73 or 5'-NT) play an important role in the genesis of asthmatic increase of airway vagal activity (8). Medullary airway vagal preganglionic neurons (AVPNs) are normally excited by extracellular ATP via P2X receptor-mediated facilitation of the excitatory inputs and a direct postsynaptic 
excitation, and inhibited by extracellular adenosine (ADO) via $A D O A_{1}$ receptor $\left(A_{D O A} R\right)$-mediated opposite actions. Allergic decreases in the expression and activity of central CD73 reduce the degradation of extracellular ATP to ADO. As a result, the excitation of AVPNs by extracellular ATP is enhanced; and the inhibition of AVPNs by extracellular ADO is weakened, leading to asthmatic increase of airway vagal activity (8). Therefore, to withstand the asthmatic increase of airway vagal activity, it might be a reasonable strategy to interfere with the downregulation of central CD73.

Statins are inhibitors of 3-hydroxy-3-methyl-glutaryl-CoA (HMG-CoA) reductase (HMGCR), the ratelimiting enzyme in cholesterol biosynthesis. These drugs are widely used in the treatment of hyperlipidemia and cardiovascular disease clinically. Both preclinical studies in animal models and some randomized clinical trials have implicated that statins also act as modulators of immune cells and are able to alleviate asthma and chronic obstructive pulmonary diseases (COPD) [for review, see (9-13)]. In particular, inhaled statins are thought to be more prospective in asthma treatment due to the assumed heavier drug deposition in the lungs (10). A latest retrospective clinical study even shows that in-hospital use of statins is associated with a reduced risk of mortality among individuals with COVID-19 (14).

The therapeutic effects of statins on cardiovascular diseases have been proved to be closely related to the upregulation of CD73 in the heart and vascular endothelium (15-21). It then becomes interesting whether statins are able to interfere with the asthmatic deceases in the expression and activity of central CD73, and, subsequently, alleviate asthma through attenuation of the asthmatic increase of airway vagal activity. Elucidation of this issue is essential to understand the therapeutic effects of statins not only on asthma/COPD but also on some other diseases with disordered autonomic function, such as cardiovascular diseases and inflammatory intestinal diseases.

This study aims to test the hypothesis that in rat model of allergic asthma, inhaled rosuvastatin is able to reverse the decreases in the central expression and activity of CD73, which attenuates the increase of airway vagal activity and, subsequently, improves pulmonary function.

\section{Materials and methods}

\subsection{Animals and ethical approval}

All of the animal procedures in this study are in accordance with the recommendations of the guidelines for the Care and Use of Laboratory Animals (1996. National Academy of Sciences. Washington, DC, USA), and were approved by the Ethical Committee of the Fudan University School of Basic Medical Sciences. Pathogen-free male Sprague-Dawley (SD) rats (seven-week old, $180 \pm 10 \mathrm{~g}$ body weight) were purchased from the Experimental Animal Center of the Chinese Academy of Science in Shanghai. The animals were fed in cages with food and water available ad libitum, and housed in an air-conditioned room $\left(22-25^{\circ} \mathrm{C}\right)$ illuminated from 07:00 to 19:00. Maximal efforts were made to minimize the number of animals and their suffering.

\subsection{Preparation of rat model of allergic asthma}

The rats were assigned into three groups: control group, ovalbumin (OVA)-sensitization group (sensitization group) and OVA-sensitization plus rosuvastatin inhalation group (treatment group). On the $0^{\text {th }}$ and $7^{\text {th }}$ day, each of the rats in sensitization group and treatment group received intraperitoneal injection of $10 \mathrm{mg}$ OVA (Sigma-Aldrich, grade V) and $2 \mathrm{mg} \mathrm{Al}(\mathrm{OH})_{3}$ suspended in $1 \mathrm{~mL}$ saline; and each of the rats in control group received intraperitoneal injections of $2 \mathrm{mg} \mathrm{Al}(\mathrm{OH})_{3}$ suspended in $1 \mathrm{~mL}$ saline. From the $14^{\text {th }}$ to $28^{\text {th }}$ or $29^{\text {th }}$ day, rats in control group, sensitization group and treatment group were challenged daily for 30 min in a closed acrylic container $(60 \mathrm{~cm} \times 40 \mathrm{~cm} \times 40 \mathrm{~cm})$ with aerosolized saline, aerosolized $5 \%$ OVA (Sigma-Aldrich, grade II, suspended in saline), and aerosolized $5 \%$ OVA and $0.4 \%$ rosuvastatin in mixture, respectively.

2.3 Plethysmography, measurement of ATP concentration in cerebral spinal fluid (CSF), Western-blot and CD73 activity assay 
All of the experimental protocols in this section have been described detailed in a previous study from this laboratory (8). Briefly, on the $29^{\text {th }}$ or $30^{\text {th }}$ day, rats were anesthetized with intraperitoneal injection of a mixture of anesthetics (urethane $0.84 \mathrm{~g} \cdot \mathrm{kg}^{-1}, \alpha$-chloralose $42 \mathrm{mg} \cdot \mathrm{kg}^{-1}$ and borax $42 \mathrm{mg} \cdot \mathrm{kg}^{-1}$, dissolved in saline), and airway vagal activity was estimated by plethysmographic measurement of inspiratory resistance of the airway $\left(R_{\mathrm{i}}\right)$, expiratory resistance of the airway $\left(\mathrm{R}_{\mathrm{e}}\right)$ and dynamic compliance of the lungs $\left(\mathrm{C}_{\mathrm{dyn}}\right)$ using a pulmonary function analyzing system (AniRes2005, Beijing Biolab Co. Ltd., Beijing, China). CSF was collected with a microsyringe punctured into the cisterna magna, and ATP concentration in CSF was measured with the luciferin-luciferase method using the Labsystems Luminoskan TL Plus luminometer (Thermo Scientific, Waltham, MA, USA) in combination with an ATP assay kit (S0026, Beyotime Biotechnology, Shanghai, China). After decapitation, brain tissue containing the NA and the middle lobe of the right lungs were isolated and put in $200 \mu \mathrm{L}$ RIPA lysis buffer (P0013C, Beyotime Biotechnology) containing a protease inhibitor cocktail for general use (P1006, Beyotime Biotechnology), and homogenized. After centrifugation, the supernatant was collected and the concentration of total protein was measured using a microplate reader (Infinite M1000 Pro, Tecan, Switzerland) in combination with a BCA protein assay kit (CoWin Biosciences, Beijing, China). Samples with equal amount of protein were mounted on a running gel of $10 \%$ or $12 \%$ SDS-PAGE electrophoresis, and transferred onto a polyvinylidene fluoride membrane (Millipore, MA, USA). After incubation in a blocking buffer (TBST containing $5 \% \mathrm{w} / \mathrm{v}$ non-fat milk), the blots were incubated with a primary rabbit antibody against CD73 (ab175396; 1:2000; Abcam, UK), eosinophil cationic protein 1(ECP1 or EAR1)(bs-1754R; 1:2000; Bioss, China) and glyceraldehyde-3-phosphate dehydrogenase (GAPDH,1:10000, 10494-1-AP, Proteintech Group, Inc., Chicago, USA), respectively, overnight at 4. This was followed by incubation with peroxidase-labeled goat anti-rabbit IgG secondary antibody (A0208; 1:2000; Beyotime Biotechnology) at room temperature for $2 \mathrm{~h}$. Specific antibody-antigen complex was detected using an enhanced chemiluminescence Western-blot detection system (P10100, NCM Biotech, Suzhou, China) and imaged using the image analyzer (TanonImage, Tanon, Shanghai, China). General protein from the NA-containing brain tissue was isolated, and CD73 activity assay was performed using a microplate reader (Infinite M1000 Pro, Tecan) in combination with a CD73 activity assay kit (A041-2, Nanjing Jiancheng Bioengineering Institute, Nanjing, China).

\subsection{Statistical analyses}

In Western-blot experiments, densitometric data were expressed as the ratio to GAPDH protein. The density ratio of CD73 to GAPDH or that of ECP1 to GAPDH obtained from all of the samples of rats in control group was averaged, and that obtained from the sample of individual rats was further divided by this average value to get the relative value. Data are presented as means $\pm \mathrm{SE}$, and compared with paired $t$-test or one-way ANOVA followed by Bonferroni correction when appropriate using the SPSS 17.0 software (SPSS, Inc., IL, USA). Shaprio-Wilk test and Kolmogorov-Smirnov test were run before parametric tests to make sure that the data follow a normal distribution. Significance was set at $p<0.05$.

\section{Results}

3.1 Inhaled rosuvastatin reversed the decreases in the expression and activity of brainstem CD73 in ovalbumin (OVA)-sensitized rats

In OVA-sensitized rats, both the expression and activity of CD73 (Fig.1A-C) in the nucleus ambiguus (NA)containing region of medulla were significantly decreased; and, consistently, ATP concentration in cerebral spinal fluid (CSF) (Fig.1D) was significantly increased. In rosuvastatin-treated rats, all of these changes were reversed by inhaled rosuvastatin (Fig.1A-D). 
A
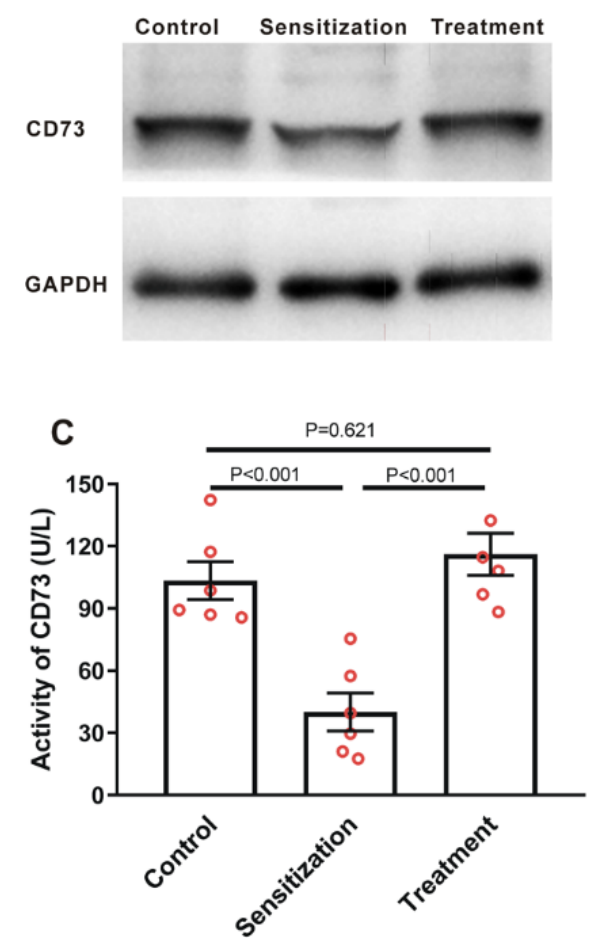
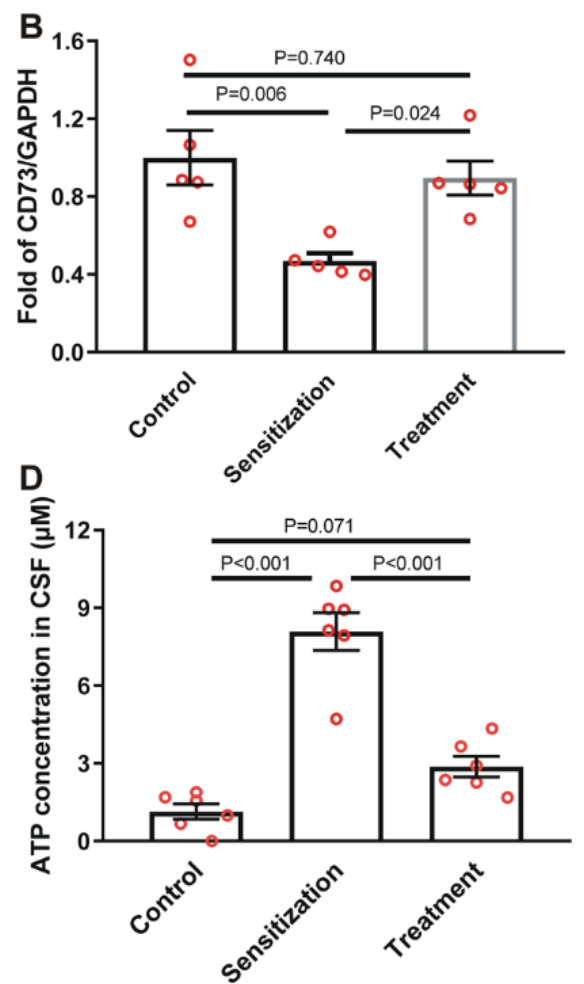

Figure 1

3.2 Inhaled rosuvastatin abolished the increase of airway vagal activity in OVA-sensitized rats

In OVA-sensitized rats, both the inspiratory resistance of the airway $\left(\mathrm{R}_{\mathrm{i}}\right)$ and expiratory resistance of the airway $\left(\mathrm{R}_{\mathrm{e}}\right)$ were significantly increased (Fig.2A1, B1), and, consistently, the dynamic compliance of the lungs $\left(\mathrm{C}_{\mathrm{dyn}}\right)$ was significantly decreased (Fig.2C1). In rosuvastatin-treated rats, $\mathrm{R}_{\mathrm{i}}, \mathrm{R}_{\mathrm{e}}$ and $\mathrm{C}_{\text {dyn }}$ are not significantly different with those of rats in control group (Fig.2A1, B1, C1; ANOVA test). 20 min after intraperitoneal injection of atropine sulphate $\left(0.5 \mathrm{mg} \cdot \mathrm{kg}^{-1}\right), \mathrm{R}_{\mathrm{i}}, \mathrm{R}_{\mathrm{e}}$ and $\mathrm{C}_{\mathrm{dyn}}$ in rats of different groups are no longer significantly different (Fig.2A2, B2, C2; ANOVA test). In addition, atropine treatment significantly decreased $R_{i}$ and $R_{e}$, and significantly increased $C_{d y n}$ in OVA-sensitized rats, but did not cause any significant change in the $\mathrm{R}_{\mathrm{i}}, \mathrm{R}_{\mathrm{e}}$ and $\mathrm{C}_{\mathrm{dyn}}$ of rats in control group and treatment group (Fig.2A3, B3, C3; paired $t$-test). 

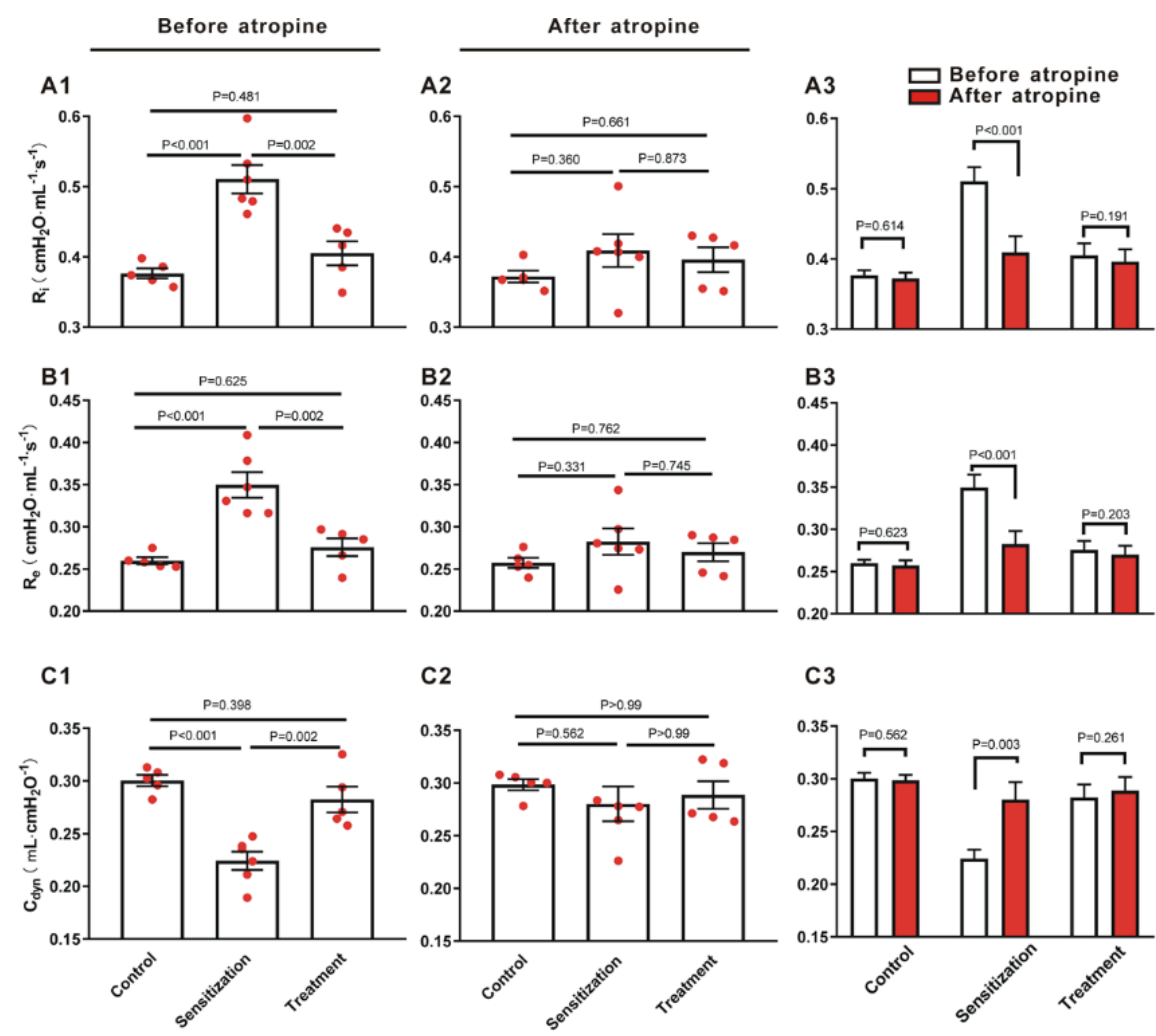

Figure 2

3.3 Inhaled rosuvastatin reversed the increase in the expression of eosinophil cationic protein 1 (ECP1) in the lungs of OVA-sensitized rats

In OVA-sensitized rats, the expression of ECP1 in the lungs was significantly increased, which was reversed in rosuvastatin-treated rats (Fig.3) (ANOVA test).
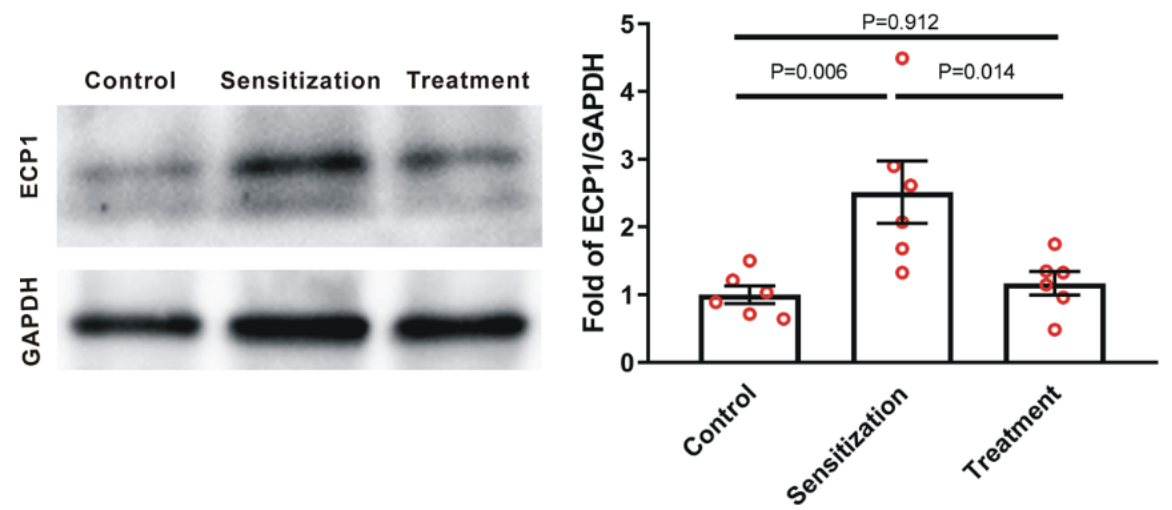

Figure 3

\section{Discussion}

There are three major new finding in this study. First, inhaled rosuvastatin reversed the decreases in the expression and activity of medullary CD73, and, consistently, reversed the increase of ATP concentration in 
CSF, in OVA-sensitized rats. Second, inhaled rosuvastatin blocked the vagal-mediated decrease of pulmonary function in OVA-sensitized rats. Third, rosuvastatin reversed the increase in the expression of ECP1 in the lungs of OVA-sensitized rats.

In this study, OVA-sensitized rats showed significant increases in $R_{i}$ and $R_{e}$, and a significant decrease in $\mathrm{C}_{\mathrm{dyn}}$, indicating decreased pulmonary function. Meanwhile, Western-blot essay showed increased expression of ECP1 in the lungs, indicating infiltration of eosinophils and pulmonary inflammation. These functional and inflammatory changes suggest a successful model of allergic asthma, as we have previously reported (22).

In this study, acute atropine treatment abolished the changes in the $R_{i}, R_{e}$ and $C_{d y n}$ of rats in sensitization group, but not in those of rats in control group and treatment group. And, after atropine treatment, the $\mathrm{R}_{\mathrm{i}}$, $R_{e}$ and $C_{d y n}$ of rats in sensitization group are not significantly different with those of rats in control group and treatment group, whether compared with the values before or those after atropine treatment. These results suggest that physiologically, airway vagal nerves might exert little tonic effect on pulmonary function; and, in an acutely or subacutely prepared animal model of allergic asthma, the decrease of pulmonary function is primarily caused by increased airway vagal activity.

In consistence with the findings of many previous studies, this study also provided evidence that inhaled rosuvastatin is able to alleviate pulmonary inflammation, as is manifested by the reversed increase in the expression of ECP1 in the lungs. However, since acute atropine treatment alone abolished the decrease of pulmonary function in OVA-sensitized rats, inflammatory changes might be poorly related to pulmonary function; and, in rosuvastatin-treated rats, alleviated pulmonary inflammation might contribute little, if any, to the improvement of pulmonary function. Our conclusions are consistent with those of previous studies that increased airway vagal activity is the major factor that induces asthma symptoms and decrease of pulmonary function $(5,7)$.

Since the pulmonary function decrease in OVA-sensitized rat is vagal-mediated, and was accompanied by decreases in the expression and activity of central CD73, the changes of central CD73 might play a key role in the asthmatic increase of airway vagal activity. Likewise, since the rosuvastatin-induced improvement of pulmonary function was accompanied by reversed expression and activity of central CD73, this reversal might play a key role in the rosuvastatin-induced attenuation of airway vagal activity. Therefore, this study for the first time provides evidence that the asthma-alleviating effect of rosuvastatin involves a central effect that attenuates the asthmatic increase of airway vagal activity, which either is mediated through a direct central action or is secondary to the modulatory action on peripheral immune cells, or involves both of these actions.

Statins have been in clinical use for decades for the treatment of hyperlipidemia and cardiovascular disease, with the tolerable side effects and relatively better affordability. In the last decade, preclinical studies in animal asthma models have obtained rather solid evidence that statins are effective both in improvement of pulmonary function and in alleviation of pulmonary inflammation. However, some small randomized clinical trials using oral statins have yielded contradictory results. While a consistent anti-inflammatory effect of statins is validated, improved clinical outcomes in terms of symptoms or pulmonary function are not demonstrated [for review, see (10)]. The results from this study suggest that in OVA-sensitized rats, the rosuvastatin-induced improvement of pulmonary function is closely related to the reversed increase of airway vagal activity. Therefore, whether statins are effective in alleviating the symptoms and decrease of pulmonary function of asthma might be determined by whether these asthmatic changes are caused by increased airway vagal activity. In human patients, asthma is known to have multiple phenotypes, such as allergic asthma, non-allergic asthma, asthma with fixed obstruction, eosinophilic asthma and neutrophilic asthma (23); and, it is unclear whether increased airway vagal activity exists in all of these phenotypes and is the major factor that induces asthma symptoms and decrease of pulmonary function. Therefore, without considering the diversity of human asthma phenotypes and the level of airway vagal activity in different phenotypes, it might be hard for randomized clinical trials to demonstrate the effectiveness of statins in alleviating asthma symptoms or decrease of pulmonary function. Airway vagal activity and/or ATP concentration in CSF might should be clinically evaluated/tested and used as indices of asthma, either in predicting the effectiveness of 
statins or in the design of new clinical trials using statin therapy. Further clinical trials in selected asthma patients with clearly increased airway vagal activity is expected.

In terms of the alleviating effects of statins on asthma symptoms and asthmatic decrease of pulmonary function, our results do not support the assumption that inhaled statins might have more advantage over those ingested or injected (10), since these effects might involve a direct central drug action that is independent of the deposition of inhaled drugs in the lungs.

Purinergic signaling plays important roles in the function of brain cells; and aberrant purinergic signaling is involved in the pathogenesis of brain injuries in many brain disorders, such as trauma, stroke, epilepsy and multiple types of chronic neurodegeneration. Meanwhile, statins have been proved to be beneficial to many brain disorders. However, it has never been reported that the beneficial effects of statins on disordered brain involve an effect on the expression and activity of CD73. This study for the first time shows that in OVAsensitized rats, rosuvastatin is able to restore the expression and activity of CD73 in the brain, and by which attenuate allergic airway vagal dysfunction. Our results implicate that in many peripheral diseases with concurrent neural disorders, the therapeutic effects of statins might also involve unrevealed central actions. For instance, increased extracellular ATP in the medullary sympathoexcitatory centers has been implicated to participate in the genesis of hypertension (24); however, it is unknown whether the anti-hypertensive effect of statins involves a central sympatholytic action through upregulation of CD73. Therefore, this study has opened a new scope for disease-based neuropharmacology of statins.

In conclusion, this study demonstrates that in rat model of allergic asthma, inhaled rosuvastatin reverses the decreases in the expression and activity of central CD73, which prevents pulmonary function decrease through abolished increase of airway vagal activity.

\section{Acknowledgments and Conflict of Interest:}

This study was sponsored by the NSFC (National Natural Science Foundation of China) grants 81770003 and 81270060 to J Wang.

The authors declare no competing financial interests.

\section{References:}

1. Yach D, Hawkes C, Gould CL, Hofman KJ. The global burden of chronic diseases: overcoming impediments to prevention and control. JAMA 2004;291(21):2616-22.

2. Liccardi G, Salzillo A, Calzetta L, Cazzola M, Matera MG, Rogliani P. Can bronchial asthma with an highly prevalent airway (and systemic) vagal tone be considered an independent asthma phenotype? Possible role of anticholinergics. Respir Med 2016;117:150-3.

3. Szekely JI, Pataki A. Recent findings on the pathogenesis of bronchial asthma. Part II. The role of hormonal predisposition, environmental influences and conditioning leading to bronchial asthma. Acta Physiol Hung 2009;96(3):289-305.

4. Lutz W, Sulkowski WJ. Vagus nerve participates in regulation of the airways: inflammatory response and hyperreactivity induced by occupational asthmogens. Int J Occup Med Environ Health 2004;17(4):417-31.

5. Liu R, Song J, Li H, Wu Z, Chen H, Wu W, et al. Treatment of canine asthma by high selective vagotomy. J Thorac Cardiovasc Surg 2014;148(2):683-9.

6. Buhl R, Hamelmann E. Future perspectives of anticholinergics for the treatment of asthma in adults and children. Ther Clin Risk Manag 2019;15:473-485.

7. Gudovskii LM, Karashurov SE, Karashurov ES, Volkov AA, Parshin VD. [Surgical treatment of bronchial asthma]. Khirurgiia (Mosk) 2002(7):14-8.

8. Chen X, Zeng M, He D, Yan X, Chen H, Chen Y, et al. Asthmatic Augmentation of Airway Vagal Activity 
Involves Decreased Central Expression and Activity of CD73 in Rats. ACS Chem Neurosci 2019;10(6):28092822 .

9. Thomson NC. Clinical Studies of Statins in Asthma and COPD. Curr Mol Pharmacol 2017;10(1):60-71.

10. Zeki AA, Elbadawi-Sidhu M. Innovations in asthma therapy: is there a role for inhaled statins? Expert Rev Respir Med 2018;12(6):461-473.

11. Al-Sawalha NA, Knoll BJ. Statins in Asthma: A Closer Look into the Pharmacological Mechanism of Action. Pharmacology 2016;98(5-6):279-283.

12. Bhattacharjee D, Chogtu B, Magazine R. Statins in Asthma: Potential Beneficial Effects and Limitations. Pulm Med 2015;2015:835204.

13. So JY, Dhungana S, Beros JJ, Criner GJ. Statins in the treatment of COPD and asthma-where do we stand? Curr Opin Pharmacol 2018;40:26-33.

14. Zhang XJ, Qin JJ, Cheng X, Shen L, Zhao YC, Yuan Y, et al. In-Hospital Use of Statins Is Associated with a Reduced Risk of Mortality among Individuals with COVID-19. Cell Metab 2020.

15. Obata T, Nakashima M. Fluvastatin, an HMG-CoA reductase inhibitor, facilitate adenosine production in the rat hearts via activation of ecto-5'-nucleotidase. Microvasc Res 2016;107:1-5.

16. Ledoux S, Laouari D, Essig M, Runembert I, Trugnan G, Michel JB, et al. Lovastatin enhances ecto5'-nucleotidase activity and cell surface expression in endothelial cells: implication of rho-family GTPases. Circ Res 2002;90(4):420-7.

17. Meijer P, Oyen WJ, Dekker D, van den Broek PH, Wouters CW, Boerman OC, et al. Rosuvastatin increases extracellular adenosine formation in humans in vivo: a new perspective on cardiovascular protection. Arterioscler Thromb Vasc Biol 2009;29(6):963-8.

18. Zukowska P, Kutryb-Zajac B, Toczek M, Smolenski RT, Slominska EM. The role of ecto-5'-nucleotidase in endothelial dysfunction and vascular pathologies. Pharmacol Rep 2015;67(4):675-81.

19. Meijer P, Wouters CW, van den Broek PH, de Rooij M, Scheffer GJ, Smits P, et al. Upregulation of ecto5'-nucleotidase by rosuvastatin increases the vasodilator response to ischemia. Hypertension 2010;56(4):7227.

20. Osman L, Amrani M, Ilsley C, Yacoub MH, Smolenski RT. Atorvastatin accelerates extracellular nucleotide degradation in human endothelial cells. Mol Cell Biochem 2008;308(1-2):209-17.

21. Osman L, Amrani M, Isley C, Yacoub MH, Smolenski RT. Stimulatory effects of atorvastatin on extracellular nucleotide degradation in human endothelial cells. Nucleosides Nucleotides Nucleic Acids 2006;25(911):1125-8.

22. He D, Chen H, Zeng M, Xia C, Wang J, Shen L, et al. Asthmatic Airway Vagal Hypertonia Involves Chloride Dyshomeostasis of Preganglionic Neurons in Rats. Front Neurosci 2020;14:31.

23. "Global strategy for asthma management and prevention: GINA executive summary." E.D. Bateman, S.S. Hurd, P.J. Barnes, J. Bousquet, J.M. Drazen, J.M. FitzGerald, P. Gibson, K. Ohta, P. O'Byrne, S.E. Pedersen, E. Pizzichini, S.D. Sullivan, S.E. Wenzel and H.J. Zar. Eur Respir J 2008; 31: 143-178. Eur Respir J 2018;51(2).

24. Wu KL, Hung CY, Chan JY, Wu CW. An increase in adenosine-5'-triphosphate (ATP) content in rostral ventrolateral medulla is engaged in the high fructose diet-induced hypertension. J Biomed Sci 2014;21:8.

Figure legends

Figure 1. Inhaled rosuvastatin reversed the decreases in the expression and activity of brainstem CD73 in OVA-sensitized rats. The expression of CD73 in NA-containing brain tissue was assayed 
with Western-blot; and the activity was measured with molecular biological methods. Note in OVA-sensitized rats, both the expression and activity of CD73 were significantly decreased, leading to significantly increased ATP concentration in cerebral spinal fluid, and all of which were reversed by inhaled rosuvastatin in rosuvastatin-treated rats. $\mathrm{P}$ values were obtained from one-way ANOVA.

Figure 2. Inhaled rosuvastatin abolished the increase of airway vagal activity in OVA-sensitized rats. (A1, B1 and $C 1$ ) The bar graphs of inspiratory resistance of the airway $\left(R_{i}\right)$, expiratory resistance of the airway $\left(R_{e}\right)$ and dynamic compliance of the lungs $\left(C_{\text {dyn }}\right)$ measured using plethysmography, showing that in OVA-sensitized rats, $R_{i}$ and $R_{e}$ were significantly increased; $C_{\text {dyn }}$ was significantly decreased, and all of which were reversed by inhaled rosuvastatin in rosuvastatin-treated rats. (A2, B2 and C2) The bar graphs of $\mathrm{R}_{\mathrm{i}}, \mathrm{R}_{\mathrm{e}}$ and $\mathrm{C}_{\mathrm{dyn}} 20 \mathrm{~min}$ after intraperitoneal injection of atropine sulphate $\left(0.5 \mathrm{mg} \cdot \mathrm{kg}^{-1}\right)$, showing that the changes induced by OVA sensitization were abolished. (A3, B3 and C3) comparison of $R_{i}, R_{e}$ and $C_{d y n}$ before and after atropine treatment in rats of different groups, showing that atropine significantly decreased $\mathrm{R}_{\mathrm{i}}$ and $R_{e}$, and significantly increased $C_{d y n}$ in OVA-sensitized rats, but did not cause any change in $R_{i}, R_{e}$ and $\mathrm{C}_{\mathrm{dyn}}$ of control and rosuvastatin-treated rats. $\mathrm{P}$ values in the left and middle columns were obtained from one-way ANOVA; $\mathrm{P}$ values in the right column was obtained from paired $t$-test.

Figure 3. Inhaled rosuvastatin reversed the increase in the expression of ECP1 in the lungs of OVA-sensitized rats . $\mathrm{P}$ values were obtained from one-way ANOVA. 